\title{
Failure Analysis for Micro-Electrical-Mechanical Systems (MEMS)
}

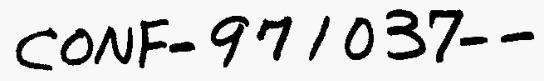

Kenneth A. Peterson, Paiboon Tangyunyong, and Daniel L. Barton

\author{
Sandia National Laboratories \\ MS1081 \\ P.O. Box 5800 \\ Albuquerque, NM 87185-1081
}

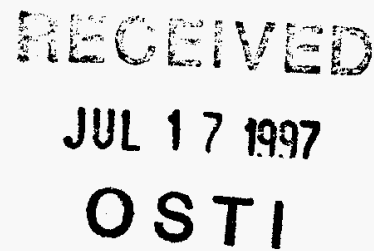

\section{Abstract}

Micro-Electrical Mechanical Systems (MEMS) is an emerging technology with demonstrated potential for a wide range of applications including sensors and actuators for medical, industrial, consumer, military, automotive and instrumentation products. Failure analysis (FA) of MEMS is critically needed for the successful design, fabrication, performance analysis and reliability assurance of this new technology. Many devices have been examined using techniques developed for integrated circuit analysis, including optical inspection, scanning laser microscopy (SLM), scanning electron microscopy (SEM), focused ion beam (FIB) techniques, atomic force microscopy (AFM), infrared (IR) microscopy, light emission (LE) microscopy, acoustic microscopy and acoustic emission analysis. For example, the FIB was used to microsection microengines that developed poor performance characteristics. Subsequent SEM analysis clearly demonstrated the absence of wear on gear, hub, and pin joint bearing surfaces, contrary to expectations. Another example involved the use of infrared microscopy for thermal analysis of operating microengines. Hot spots were located, which did not involve the gear or hub, but indicated contact between comb structures which drive microengines. Voltage contrast imaging proved useful on static and operating MEMS in both the SEM and the FIB and identified electrostatic clamping as a potentially significant contributor to failure mechanisms in microengines. This work describes MEMS devices, FA techniques, failure modes, and examples of FA of MEMS.

\section{Introduction}

MEMS are electrical-mechanical structures fabricated on silicon wafers using variations of integrated circuit processing techniques. MEMS consist of features that are fabricated using multiple polysilicon layers which are freed simultaneously from encapsulating glass in a step called the release etch. Micrometer-sized features are achievable, which can be configured to do mechanical work or serve as sensor elements. The FA outlined in this paper was performed on microengines and prototype accelerometers. The fabrication and design of the microengine we examined has been described in detail earlier [1]. A top view optical micrograph of a wirebonded microengine is shown in Figure 1.

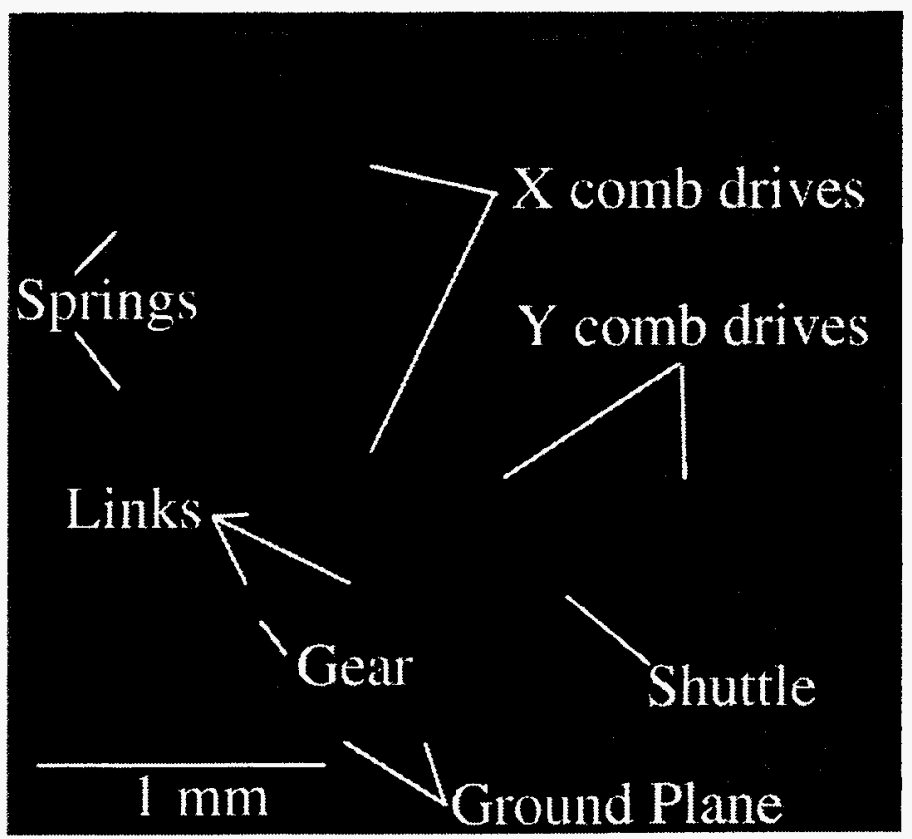

Figure 1. Wirebonded microengine (optical image).

This microengine is actuated by two orthogonal sets of electrostatic comb drives, each axis having bi-directional drive capability. The shuttles on the comb drive can be thought of as the spines of the movable combs. They connect to link arms either with a flanged pin joint or a flexible joint. A link arm connects, in turn, to the gear through a pin joint. The comb 


\section{DISCLAIMER}

This report was prepared as an account of work sponsored by an agency of the United States Government. Neither the United States Government nor any agency thereof, nor any of their employees, make any warranty, express or implied, or assumes any legal liability or responsibility for the accuracy, completeness, or usefulness of any information, apparatus, product, or process disclosed, or represents that its use would not infringe privately owned rights. Reference herein to any specific commercial product, process, or service by trade name, trademark, manufacturer, or otherwise does not necessarily constitute or imply its endorsement, recommendation, or favoring by the United States Government or any agency thereof. The views and opinions of authors expressed herein do not necessarily state or reflect those of the United States Government or any agency thereof. 
fingers are $2.4 \mu \mathrm{m}$ wide with a $3 \mu \mathrm{m}$ space between them, and are suspended about $1 \mu \mathrm{m}$ above the surface. Slender integral polysilicon spring elements are used to apply a balancing force during the drive cycle and to suspend the moving shuttle. The springs also return the gear to the equilibrium position when it is at rest. The equilibrium position occurs when the $\mathrm{X}$ shuttle is fully extended, and the pin connecting the $\mathrm{X}$ link to the gear is directly below the hub in the orientation shown in Figure 1. The areas under the combs and the gear are grounded planes. The gear, $50 \mu \mathrm{m}$ in diameter, turns on a flanged hub. A FIB cross section of an unreleased gear and pin joint is shown in Figure 2. This FIB section also illustrates what will become the equilibrium position. Each gear also has three dimples on the bottom surface to mitigate tilting during operation which can arise due to design tolerances on the spacings between the gear inner diameter and the hub outer diameter. The dimples also reduce the available area for sticking of the gear to the substrate. The shuttle and ground planes have a direct connection to electrical ground. The gear on the hub is also grounded indirectly by contact with the hub and with links. The links are grounded indirectly by contact with the gear and with their respective shuttles. A signal of $60-90 \mathrm{~V}$ is applied to the appropriate fixed comb in a sequence which attracts the grounded, moveable comb to effect motion of the shuttle which results in rotation of the gear. Research [2, 3] has resulted in optimized drive signals, and the microengines can be operated well above $100,000 \mathrm{rpm}$ for extended periods of time.

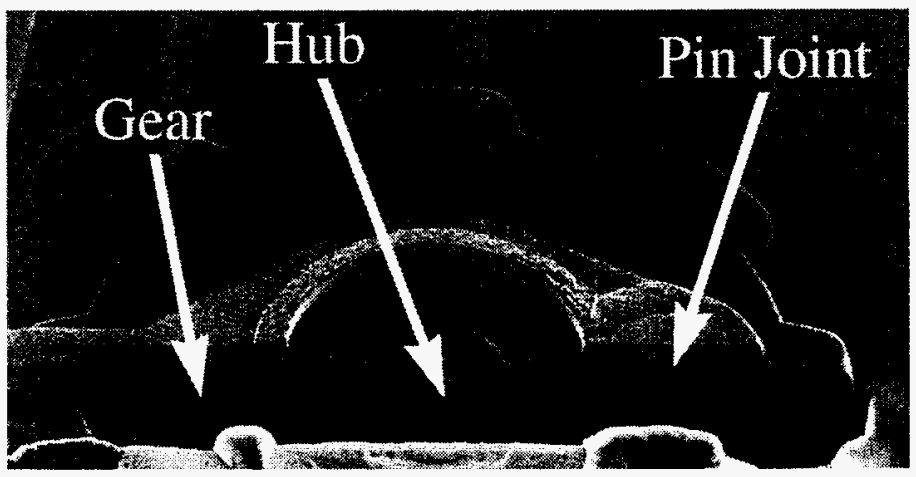

Figure 2. FIB section of unreleased gear showing hub and pin joint.

Several references to FA of micromachined moving parts have been made in the literature [4-8]. These references include instances where the SEM was used both to identify wear (debris and particles) as well as the absence of physical indications of wear. The small scale and the materials used for these features indicate that several common microelectronic FA techniques are applicable. This work goes beyond secondary electron imaging in the SEM to apply several other useful FA techniques to the analysis of MEMS.

\section{Failure Analysis Techniques}

Microengines were submitted for FA after endurance tests of up to three weeks duration in which the performance of the engines had degraded. More recently, larger numbers of microengines have been tested in a reliability evaluation [9], from which degraded engines are also being submitted for FA. These microengines were actuated during FA by applying four half sine waves of variable amplitude and frequency in 90 degree phase steps to the appropriate fixed combs of the electrostatic comb drives.

Optical Microscopy Optical observation of failed engines provides the initial verification of the failure and a great deal of other information in many cases. Debris, textures, stains, broken elements, laterally displaced elements and variations from the equilibrium position are readily observable. For example, the broken spring in Figure $3 \mathrm{a}$ is clearly visible from optical inspection. However, more subtle changes in the MEMS elements, as shown for an overdriven spring in Figure $3 \mathrm{~b}$ are also visible optically. This precursor to failure is due to arcing or attachment to the adjacent powered line, which was subsequently broken free during the microengine cycle. Close examination of all movable parts can reveal exactly which connections might suffer sticking and which are free. For example, one pinned link may be able to reciprocate with a displacement equal to the tolerance of the pin joint, while the link to which it is pinned does not move. Videotapes of operating engines have also proven useful to track performance during tests of operating life [9].
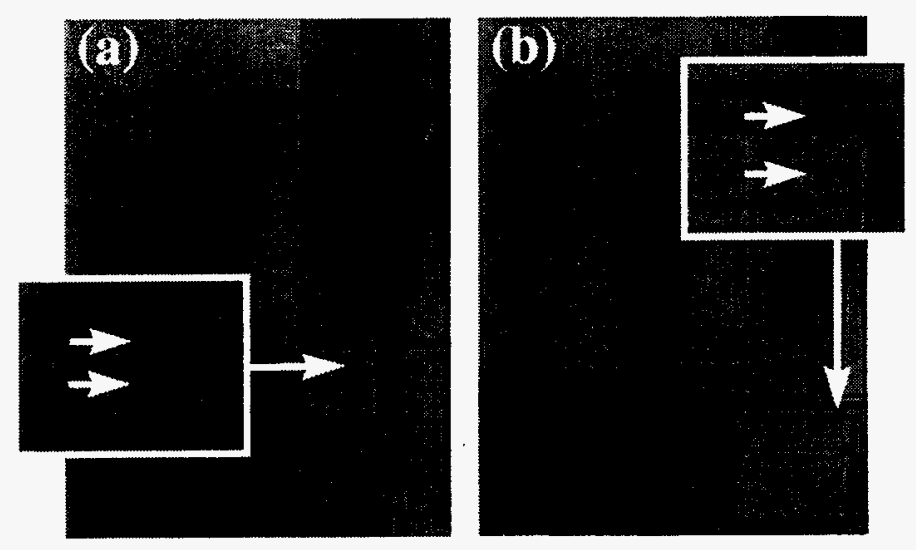

Figure 3. Low magnification optical images of degraded engines, with insets enlarged. (a) Engine with broken spring and debris from broken attachment. (b) Engine with debris due to less severe broken attachment.

Scanning Laser Microscopy (SLM) The scanning laser microscope (SLM) has been used to obtain confocal images. Confocal imaging helps resolve elements which have abnormal vertical displacements by limiting the depth of focus of the optical microscope during a particular scan. This has proven to be valuable in identifying out of plane attachments. An example is shown in Figure 4, where the out of plane stuck finger is 
clearly resolved in an image where height above the substrate is represented by false color (shown here in grayscale). The dark contrast of the tip of the finger indicated by the arrow shows that it is closer to the substrate than the adjacent fingers. This finger is in a set of fixed fingers that received a high voltage signal. A fixed finger which is stuck to the electrically grounded plane beneath it would be expected to interfere with the operation of the engine.

Scanning Electron Microscopy (SEM) The SEM has been useful for imaging defects at high magnification as well as to determine electrical continuity in static and operating microengines. Figure 5 shows a conventional use of secondary electron imaging in the SEM to identify a spring element which is in contact with the substrate (ground plane) at its mid-span. A suspended spring is shown for comparison. This would be expected to exhibit a purely mechanical effect, since both spring and ground plane are electrically grounded.

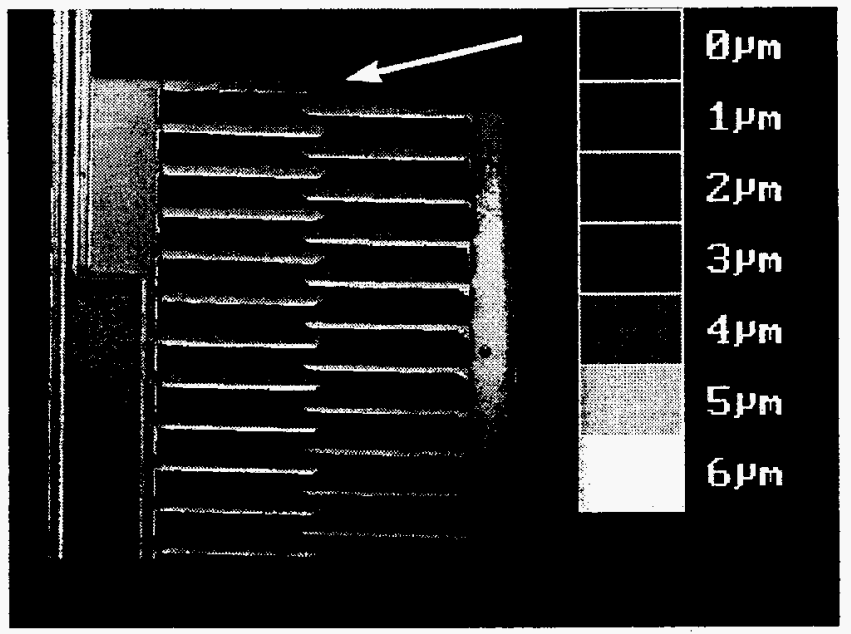

Figure 4. Confocal image showing finger which is displaced out of plane (arrow, darker contrast) and is in contact with the substrate.

Secondary electron imaging in the SEM was helpful in identifying the only wear track ever identified on the microengine, as shown in Figure 6. This wear track is likely to have resulted from an anomaly involving the particle shown at the left. The non-concentric track could only have been made by a gear/hub assembly which had a broken point of attachment to the substrate (shown at center). In another example, Figure 7 shows the comb finger imaged in Figure 4 to be in contact with the substrate. Electrical measurements indicate that the comb shorted to ground through a $9 \mathrm{k} \Omega$ path and yet, when this finger was lifted with a xenon laser cutter, the short persisted. This short has not been pinpointed by FA techniques employed to date.
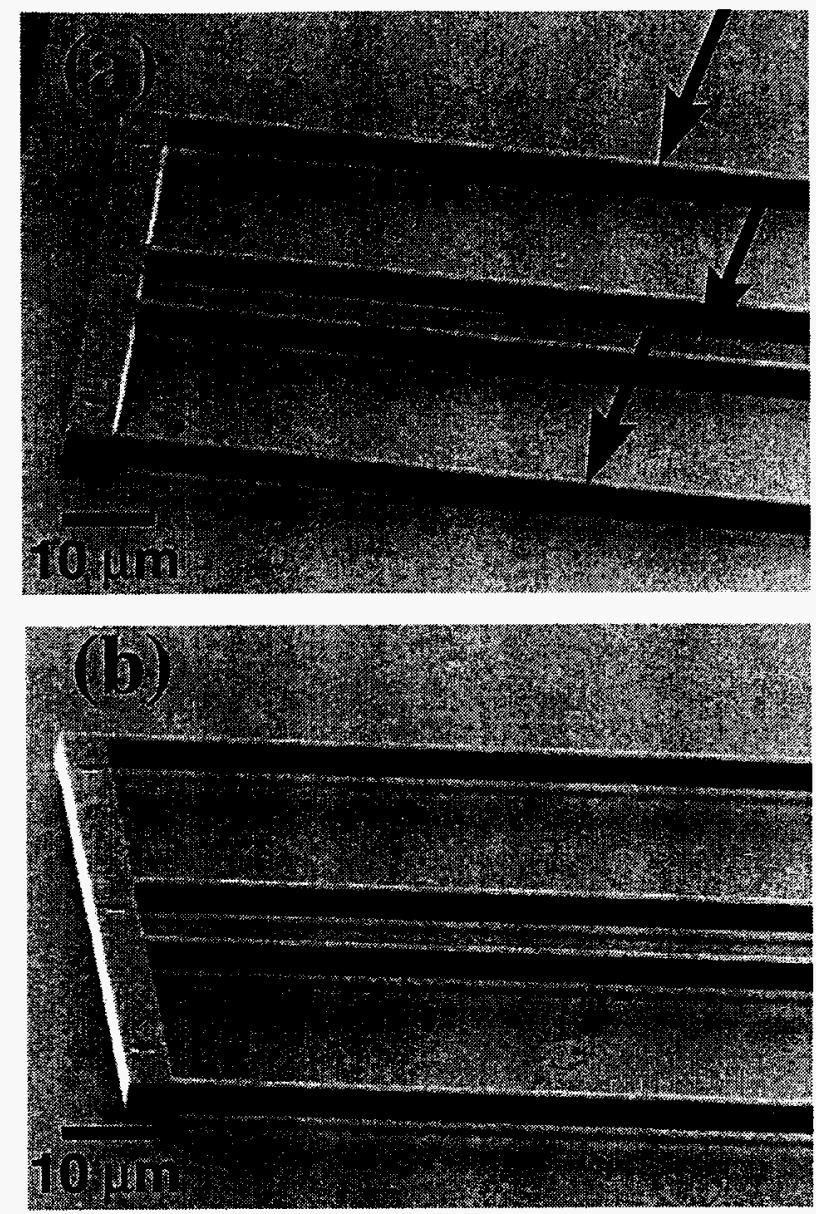

Figure 5. SEM image of spring elements. (a) Defective spring (arrows show points of contact with substrate). (b) Spring which is properly suspended above the substrate.

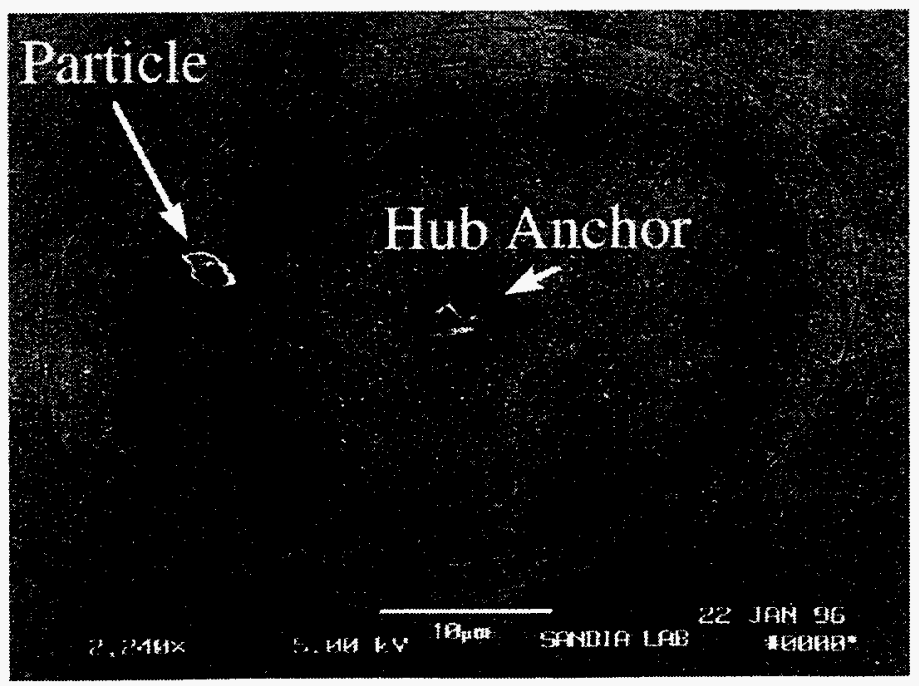

Figure 6. SEM image of a wear track related to a particle on the substrate. Concentric circles show the relationship of the track to the gear geometry. Note that the hub attachment is fractured (shown at center). 


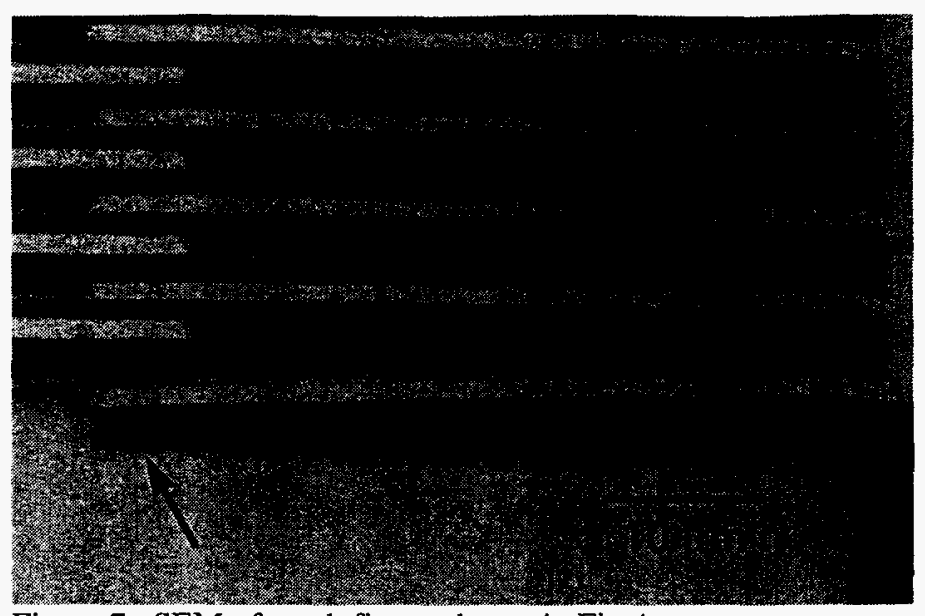

Figure 7. SEM of stuck finger shown in Fig 4.

Voltage contrast (passive and active) Passive and active voltage contrast techniques have identified structures at potentials different from those expected. Passive voltage contrast is defined as contrast which arises from voltage differences induced by rastering the beam, when various elements reach an equilibrium potential through self-charging. Active voltage contrast is defined as that arising from external selection of voltage differences on different structures. In both cases, contrast is generated by differences in secondary electron emission yields caused by differences in potential. When imaging with a primary beam energy of $1 \mathrm{keV}$, areas that are at positive potential appear dark in the images and those that are at ground appear bright. It has been possible, by increasing the accelerating potential, to observe a polarity change in the features which were charging, due to a crossover in the secondary electron emission efficiency. Figure 8 shows a gear and link arms with pin joints. The passive voltage contrast shows that the electrically floating arms and gear are charged positively (dark) with respect to the gear hub and shuttle, which have direct contact to ground. It is also evident that the engine is not in it's equilibrium position (shown in Figure 2) due to a fault. The passive voltage contrast image of Figure 9 shows a microengine with flexible links which provide a direct connection to ground through the shuttle and springs. In this case, the indirect contact is between the gear and link at a pin joint and between the gear and its hub. In this case, only the gear is charged positively with respect to the grounded elements. No performance statistics are available at this time comparing the operation of the flexible link to that of the pin joint, so the significance of this charging behavior is unknown.

No voltage contrast difference was observed between the finger and the ground plane in Figure 7, because of a $9 \mathrm{k} \Omega$ short between the conductors, as mentioned earlier. Even though the finger appeared to be in physical contact with the ground plane, that was not the source of the short. This may be due to an insulative native oxide. Another use of passive voltage contrast in the SEM was to localize defects in polysilicon interconnect lines in prototype integrated MEMS accelerometers, where CMOS circuits are integrated with the mechanical structures on the same IC. Another example of physical contact which does not constitute electrical contact is shown in Figure 10 where opposing comb fingers of an accelerometer are in contact, but not at the same potential. Figure 11 shows voltage contrast between opposing sets of combs which was not seen in control samples (all pins on the device were grounded). The dark comb fingers are charging positively at a beam energy of $1 \mathrm{keV}$ as though disconnected from ground. Tracing the interconnect for the comb led to a region of defective line containing the open shown in Figure 12. This was a condition affecting a process lot of these devices. Subsequent lots of this device showed no such opens.

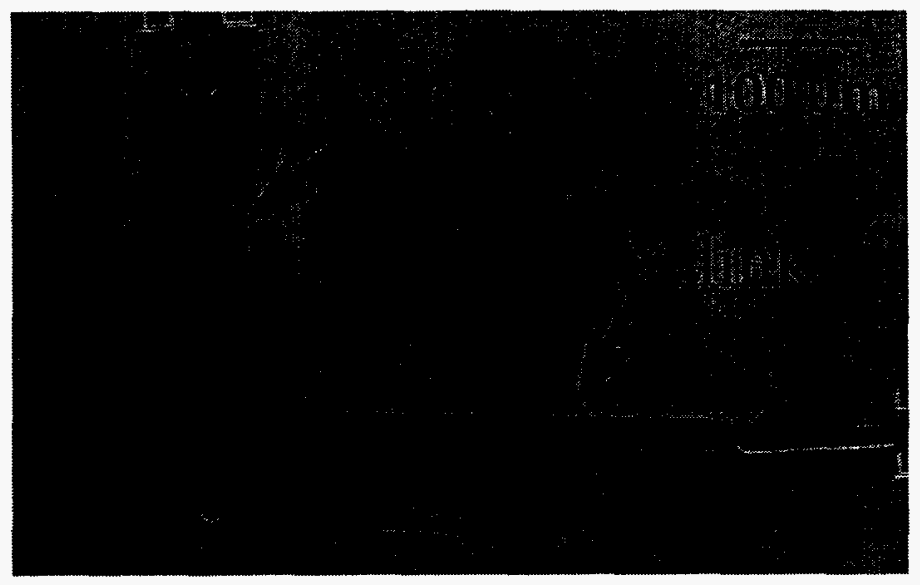

Figure 8. Passive voltage contrast showing charging of link arms and gear at $1 \mathrm{keV}$ in the SEM.

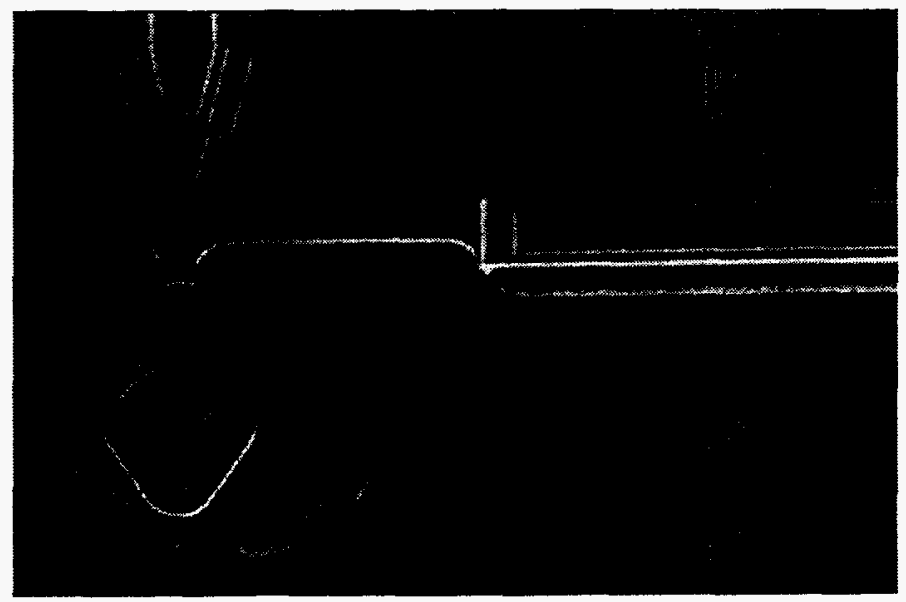

Figure 9. Passive voltage contrast showing charging of the gear only with respect to ground plane, hub, and link arms at $1 \mathrm{keV}$ in the SEM. Arrow indicates flexible link.

Operation in the SEM The same electrical feed-through capability which permits active VC imaging has been used to successfully operate microengines in the SEM. Some 
microengines stopped running during and following electron beam irradiation, while others were operated for hours with no attempt to limit beam exposure. During the operation of one such engine, the electrically charged gear and pin-jointed links were seen to discharge once per revolution. The ground plane contrast was also seen to change once per revolution. This change in VC behavior can be attributed to the intermittent contact between grounded and powered features in the microengine that occurs during the engine's operating cycle. The VC shift on ground planes has also been observed in the focused ion beam (FIB).

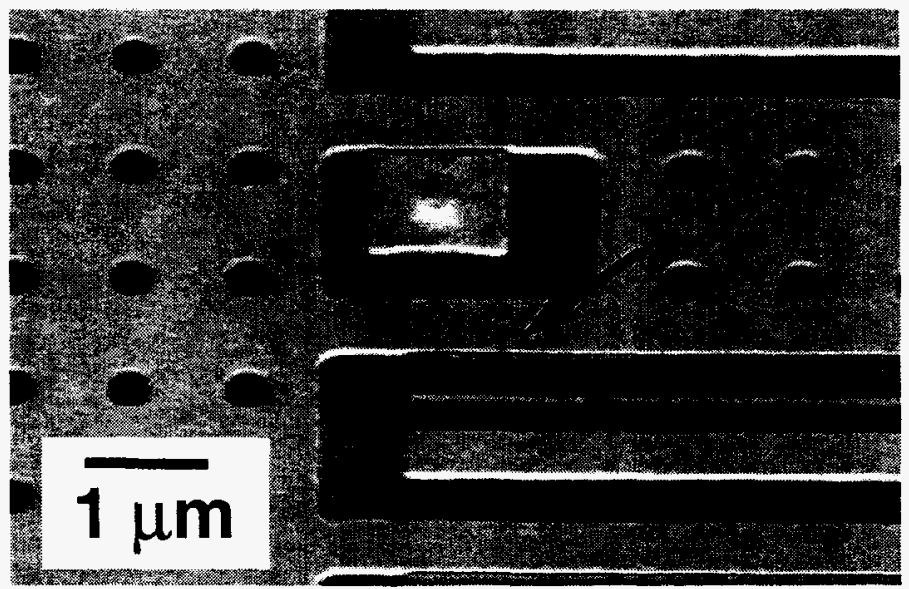

Figure 10. Electrically isolated fingers in physical contact.

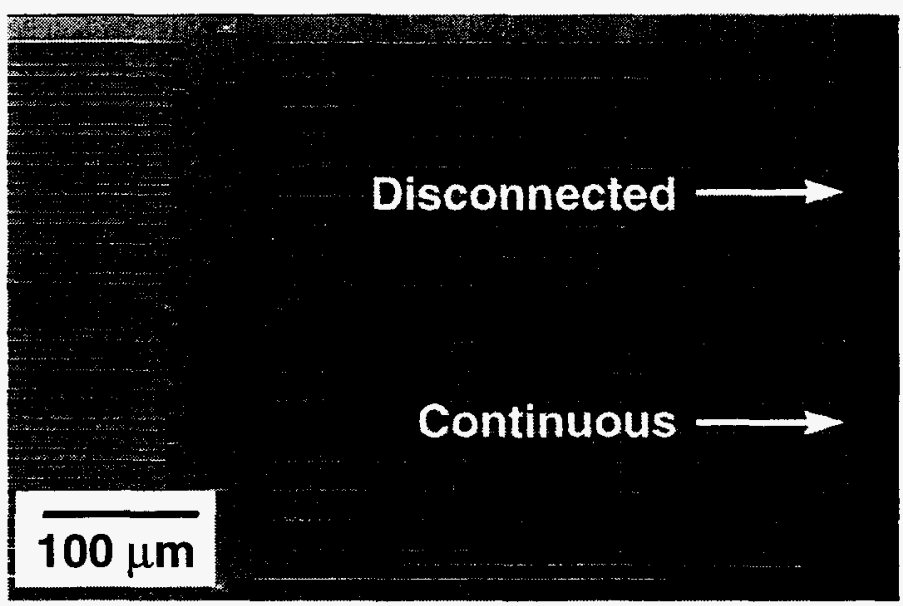

Figure 11. Voltage contrast between comb fingers at different potentials.

Focused Ion Beam (FIB) The focused ion beam (FIB) equipment uses a $30 \mathrm{keV}$ beam of positive gallium ions $(\mathrm{Ga}+)$ to irradiate the surface of the sample in a defined area. This irradiation causes surface charging, which can be neutralized by a flow of low energy $(-30 \mathrm{eV})$ electrons from a flood gun. Imaging using a signal consisting of either secondary ions or secondary electrons is possible in the FIB. The FIB has been used to image structures, to cross section elements of concern (as seen in Figure 2) and to cut elements free for subsequent examination. One interesting result is the observation of motion of the structures during irradiation by the ion beam. Figure 13 shows a gear which, as submitted for FA, behaved as though stuck on the hub. The gear moved freely, however, after initial FIB examination using charge neutralization.

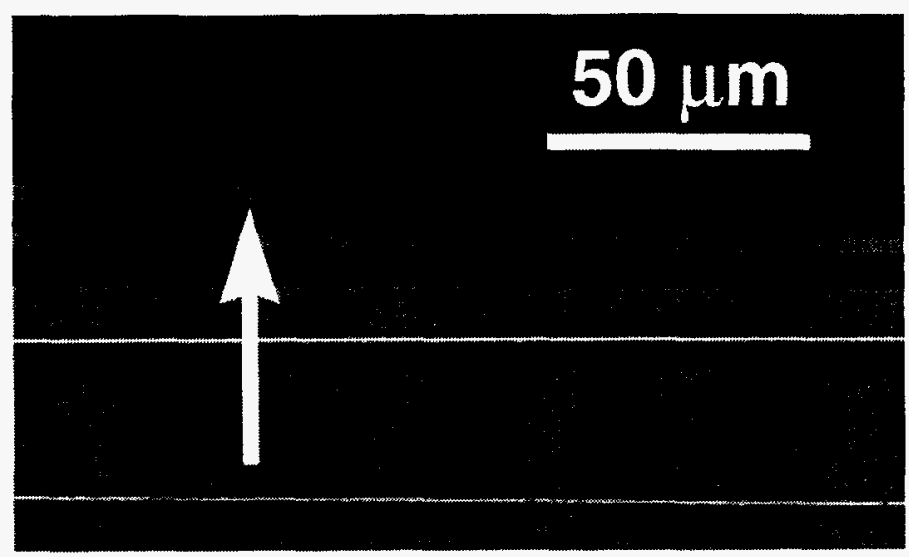

Figure 12. Open in defective polysilicon interconnect line on prototype sensor, causing the electrically floating set of combs in Figure 11.

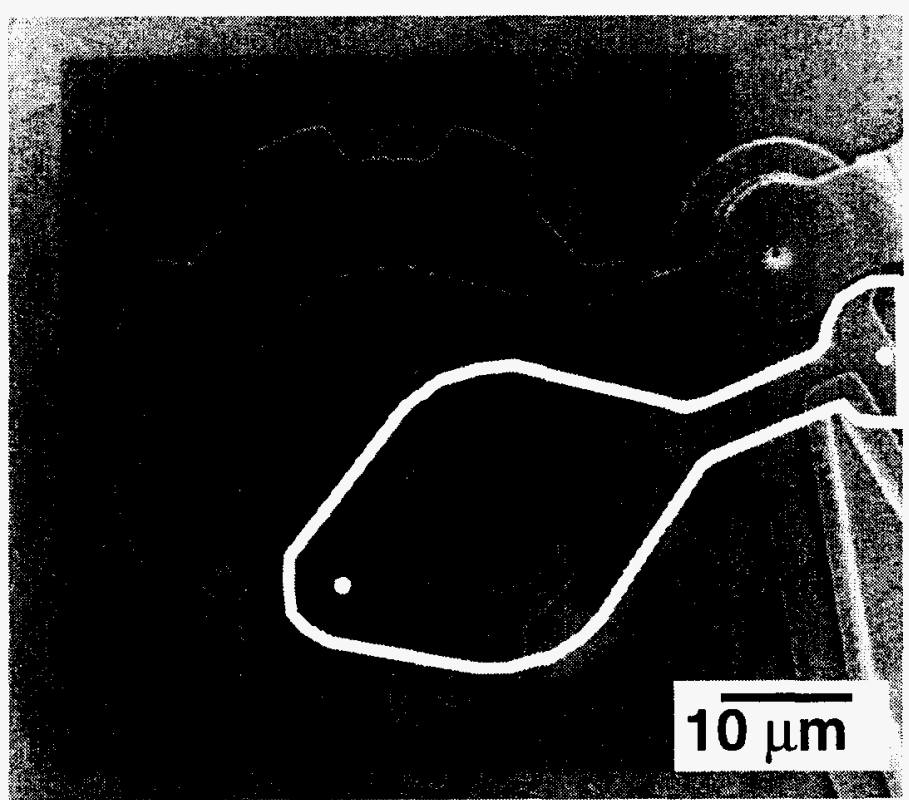

Figure 13. Evidence of the effect of charge from the ion beam on MEMS structures. The "stuck" position of the $X$ link is outlined in white.

FIB sectioning has been used to differentiate a physical condition of a stuck pin joint from that of a free pin joint. Figure 14 shows the head of the pin on the stuck pin joint to be in intimate contact with the link to which it is pinned. The free pin joint in Figure 15 shows no such contact. The plane of the cross 
section of Figure 14 intercepts less of the pin than in Figure 15, which accounts for the appearance that it is thinner.

Another use of FIB milling is to gain access to bearing surfaces which are normally hidden from view. By careful microsectioning, gears and pins have been separated. Using fine probes at a probe station, it has been possible to mechanically nudge elements to orientations where they can be carefully examined in the SEM as shown in Figure 16. This figure shows one half of a separated gear that has been flipped over to examine the back surface and the other half remaining upright but separated from the hub. This operation required extreme care, as the slightest vibration, or the existence of static charge caused these small pieces to be ejected from the sample.

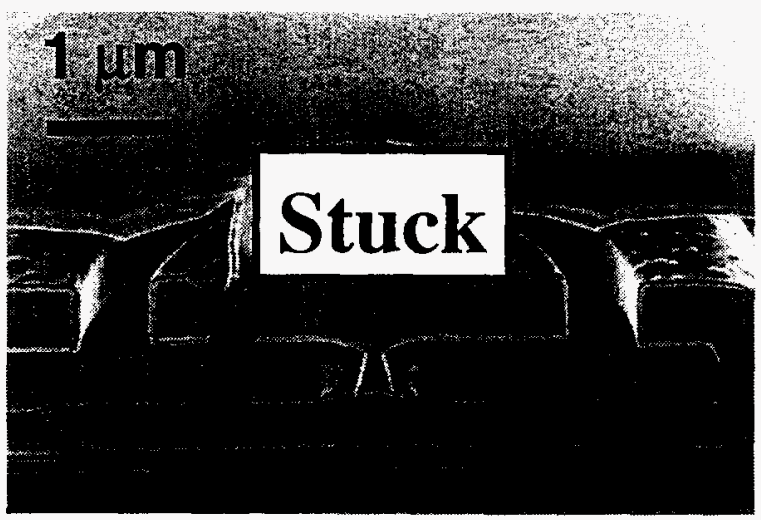

Figure 14. FIB cross section of a stuck pin joint as released.

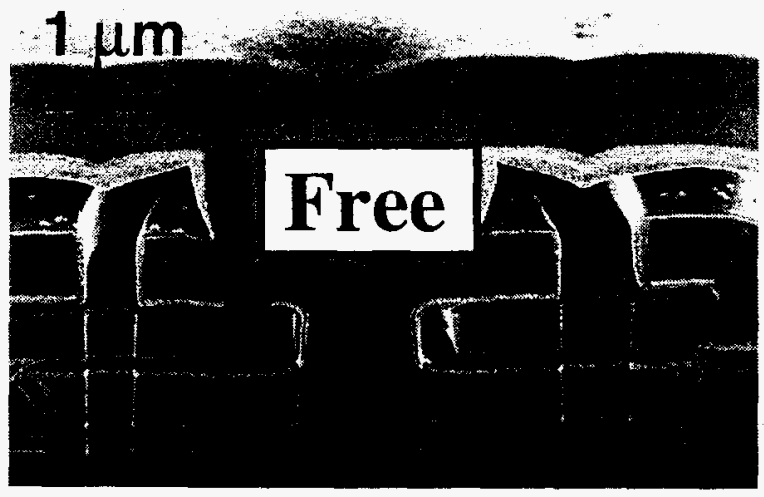

Figure 15. FIB cross section of a free pin joint as released.

After separation, the bearing surfaces of the gears were carefully examined in the SEM. Figures 17 and 1.8 show the front and back bearing surfaces of a gear which was operated until failure due to non-uniform rotation. These show a typical result which is the lack of physical indications of wear. While artifacts were observed on certain bearing surfaces, similar artifacts were seen on microengines which were never operated. The intermittence of operation of this engine can not be associated with any evidence of physical wear.

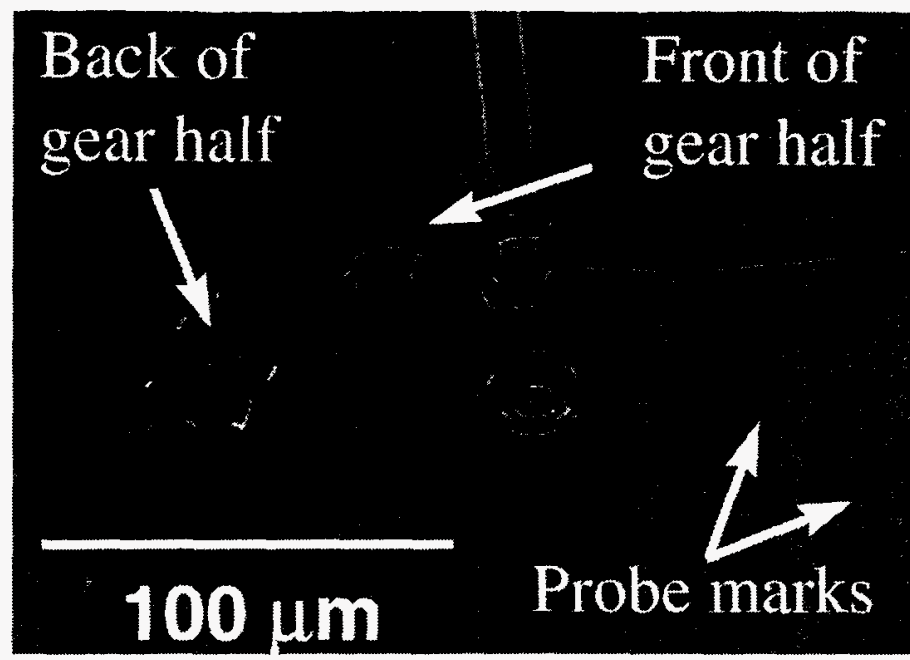

Figure 16. Microengine gear which has been microsectioned in the FIB and separated for SEM examination of bearing surfaces. Note marks made by probes.

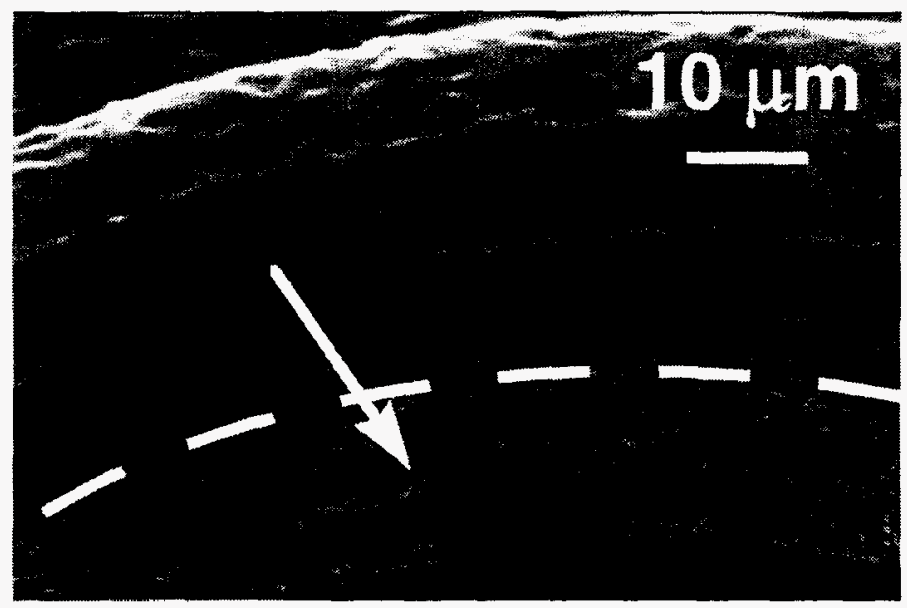

Figure 17. Top and inner surface of gear operated to failure. Arrow and dashed line indicate bearing surfaces.

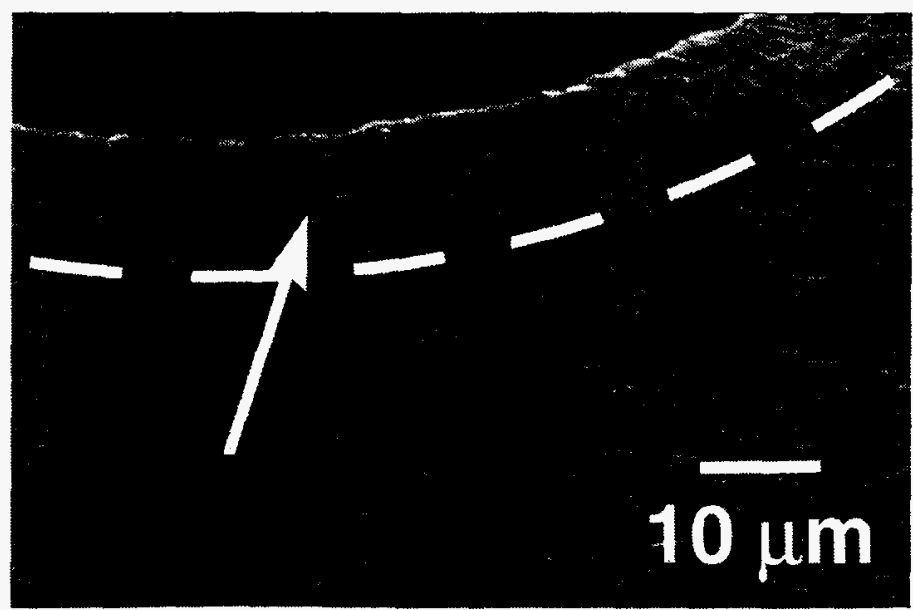

Figure 18. Back surface of gear operated to failure. Arrow and dashed line indicate bearing region. 
In an attempt to generate wear, a sample was run in a rocking mode, illustrated in Figure 19. The $X$ comb was used to push unusually hard in one direction against the hub, while the $Y$ comb was reciprocated to create a rocking motion, without completing a revolution. The rocked and not-rocked bearing surfaces were then separated in the FIB and examined for signs of wear. Not only did the opposite sides of the gear lack signs of wear but, as Figure 20 shows, the hub surfaces examined in the SEM showed no differences in wear when examined at a high tilt angle. Other edges were examined as well, to cover the possibility of contact resulting from tilting of the gear during actuation, but no wear was observed.

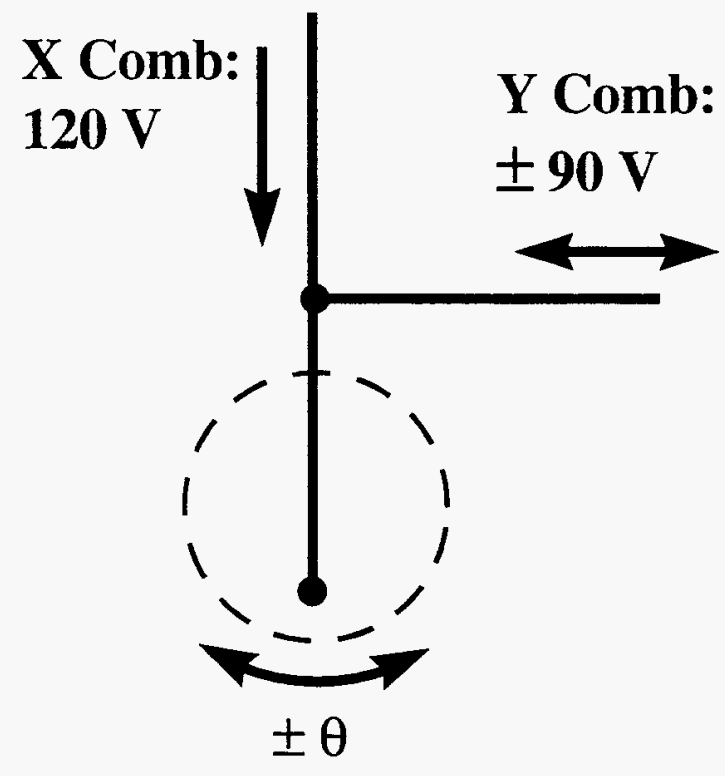

Figure 19. Schematic of rocking experiment.
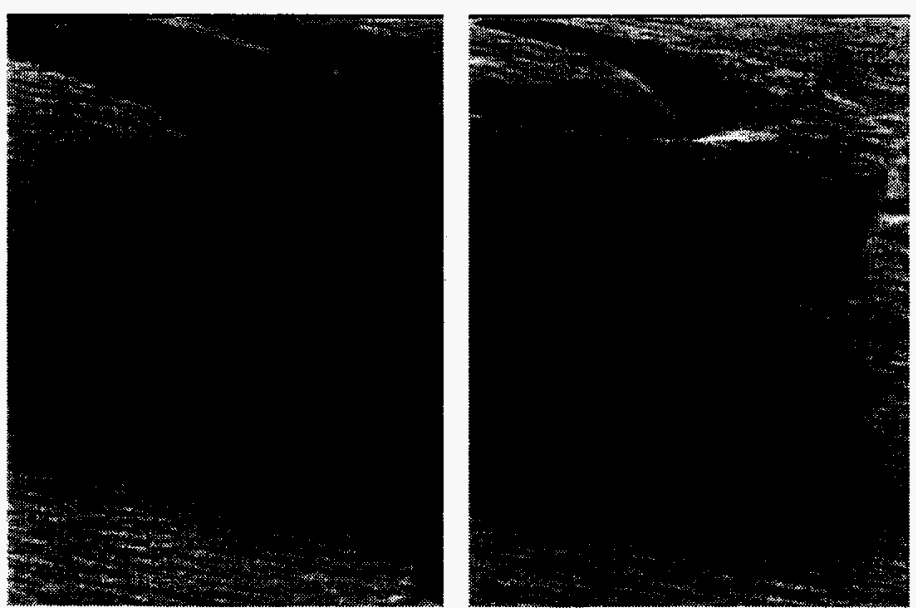

Figure 20. Hub bearing surfaces from a rocking motion experiment. Left side saw heavy pressure, right side experienced only incidental pressure.

Voltage contrast in the FIB has been studied for integrated circuits [10]. Passive and active voltage contrast imaging, without charge neutralization, has also been useful on MEMS.
Several examples involved structures in mechanical contact that were not in electrical contact. Passive voltage contrast accounts for the dark appearance of the gear in the cross section of Figure 2 , as it is electrically isolated in encapsulating glass and thus acquires a positive charge.

One complication from using the FIB has been observed. Sputtered material can redeposit from the area being milled in a line of sight fashion on the undersides of suspended members. This can create the appearance of a separate layer, such as the light contrast layer visible in FIB sections of Figures 14 and 15. This material is thickest at the milled edge, tapering back into the sample. Because this redeposited material is masked from the bearing surface by the flange of the hub, it might be confused with a wear track on the gear. An example of this is shown in Figure 21.

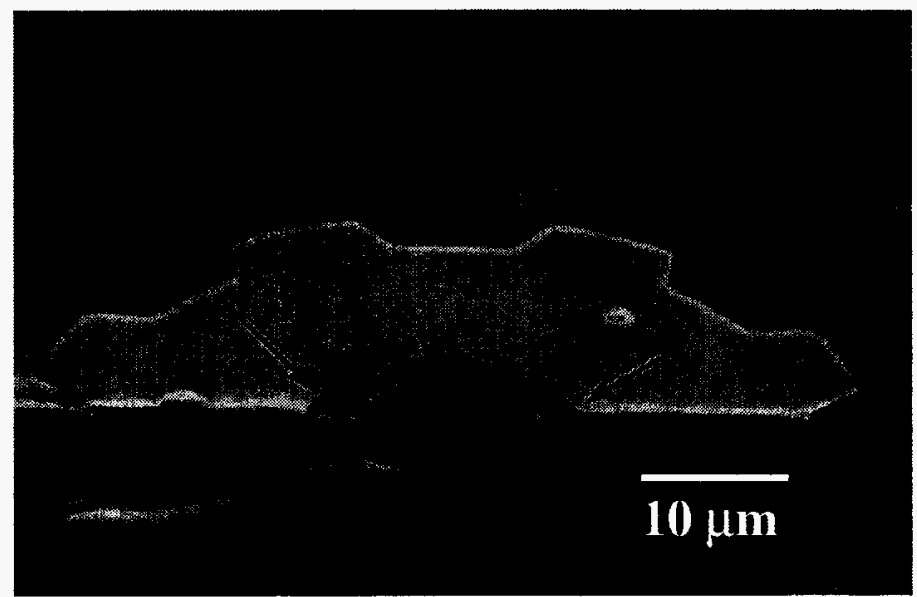

Figure 21. Micro-sectioned gear bottom surface showing FIB re-deposited material (arrows)

Atomic force microscopy The atomic force microscope provides very detailed topographic images and surface traces. The atomic force microscope was used to study the surface roughness of the wear track shown in Figure 6. Figure 22 shows a topology image of an area which includes the wear track. Smoothing was observed in the track, compared to the region outside the track, as might be expected. A line scan across the track is shown in Figure 23, indicating the ability to resolve the track against the roughness of the sample in general.

Infrared Microscopy Several operating microengines were examined using an infrared microscope to construct thermal images based on the infrared radiance emitted from the structures. Normally, temperature would be deduced from differences in emissivity using this technique, but movement in the structure which occurred when the temperature was changed to create an emissivity map of the surface and calibrate the system prevented this. The apparatus uses contrast with 0.003 $\mathrm{mW} / \mathrm{cm}^{2}$-ster per step to display differences in radiance. One finding was that for one of the engines which was operating, but rotating irregularly with a pause in the rotation cycle, hot spots (areas where the radiance increased in the image) were associated with the $X$ comb drives. No such hot spots were associated with the $\mathrm{Y}$ comb drives, nor were hot spots associated 
with comb drives of engines operating with smooth rotation. An example is shown in Figure 24. In gray scale, the hot spots appear dark, and are indicated by arrows in Figure 24. An image for the same engine at rest is shown in Figure 25. These hot spots are presumed to be associated with arcing or momentary contact of the combs. In addition, the gear and hub area were closely examined to see if frictional heating could be resolved. No increase in radiance was seen in the area of the bearing surfaces of operating engines.

\begin{tabular}{|l|l|l|}
\hline Site 1 & $\begin{array}{l}\text { Site } 2 \\
\text { Roughness } \\
\text { (RMS): } \\
125 \AA\end{array}$ \\
Roughness & $\begin{array}{l}\text { Site 3 } \\
\text { (RMS): } \\
52 \AA\end{array}$ \\
\hline $20 u g h n e s s$ \\
(RMS): \\
$83 \AA$
\end{tabular}

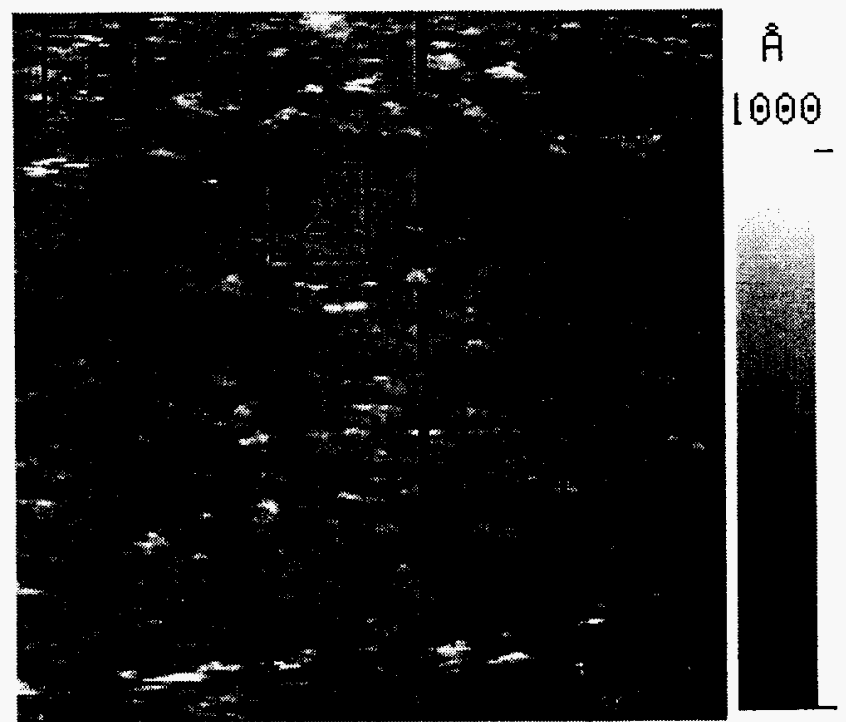

Figure 22. AFM topology image of wear track. The dashed line indicates the position of line scan of Figure 23.

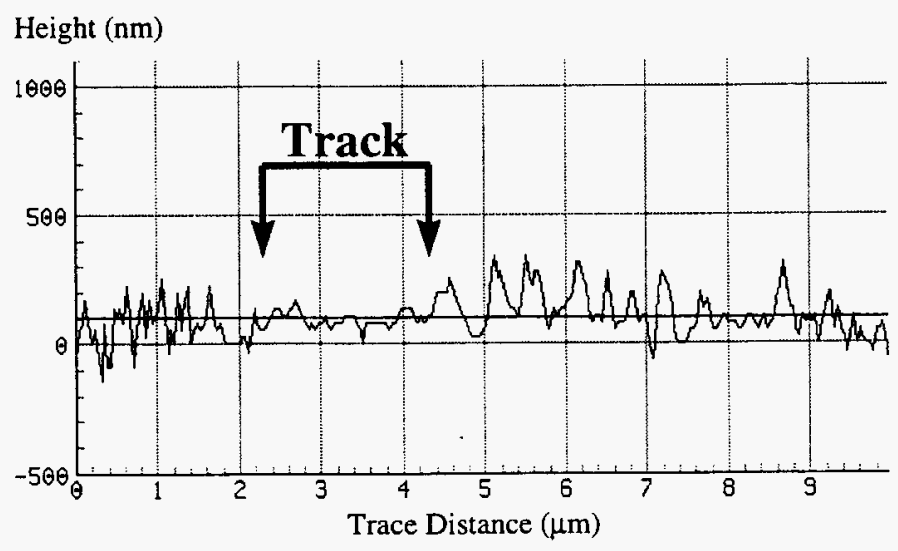

Figure 23. Topology line scan across wear track in Figure 22.

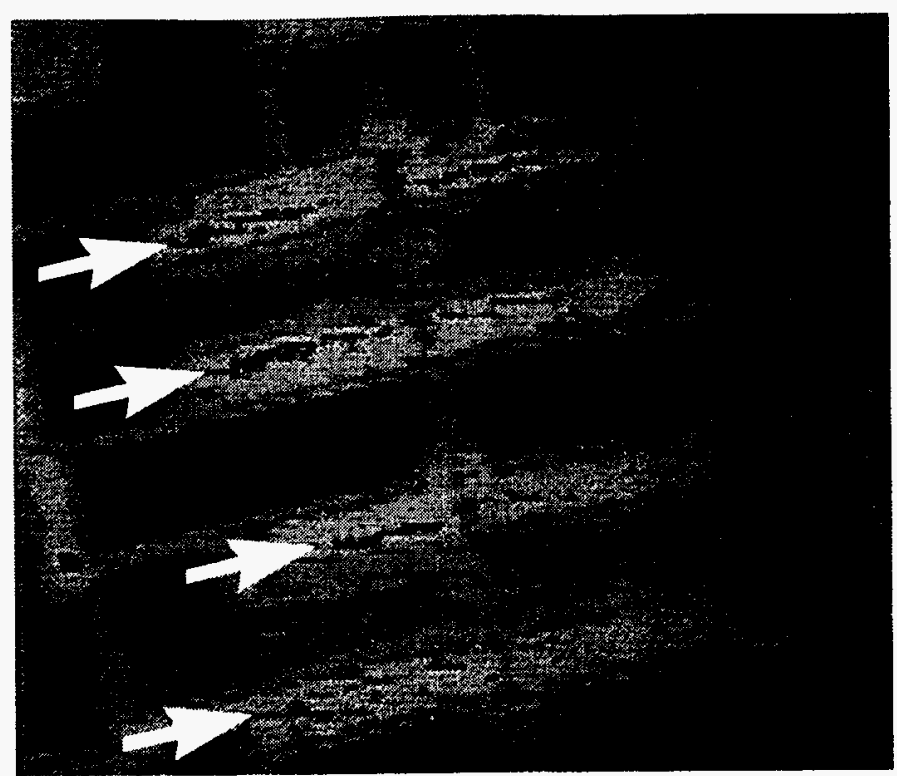

Figure 24. Infrared image of defective operating engine showing hot spots (dark in appearance) in X comb drive area.

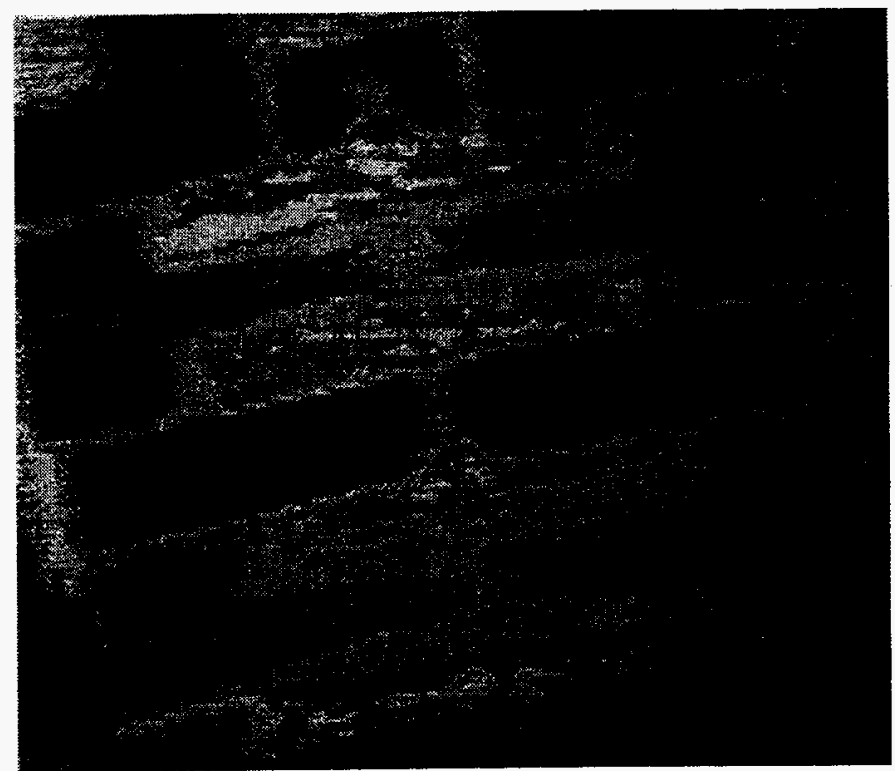

Figure 25. Infrared image of same engine at rest.

Light emission The possibility of arcing at combs suggested the use of light emission analysis, which is common in FA of ICs. The same microengine which exhibited the hot spots in Figure 24 was analyzed using LE, but no emitted light was resolved under the same operating conditions.

Acoustic Microscopy Acoustic microscopy was employed on stationary microengines in an attempt to resolve contact between stuck gears and links and the substrate. This technique is more destructive to the sample due to the introduction of water as a coupling fluid. The acoustic signal could not be translated into evidence of sticking, although morphological features were resolved. Figure 26 shows a sample of the visual output for a gear which had tilted, causing a gradient in its intensity across the image. Combs were also 
resolved, but no information was gained regarding points of contact.

Acoustic Emission Acoustic emission was also evaluated on several engines while they were running. This involved the attachment of a sensor to the package containing the microengines and "listening" for acoustic events as microengines were operated. No signal was resolvable above the background noise level.

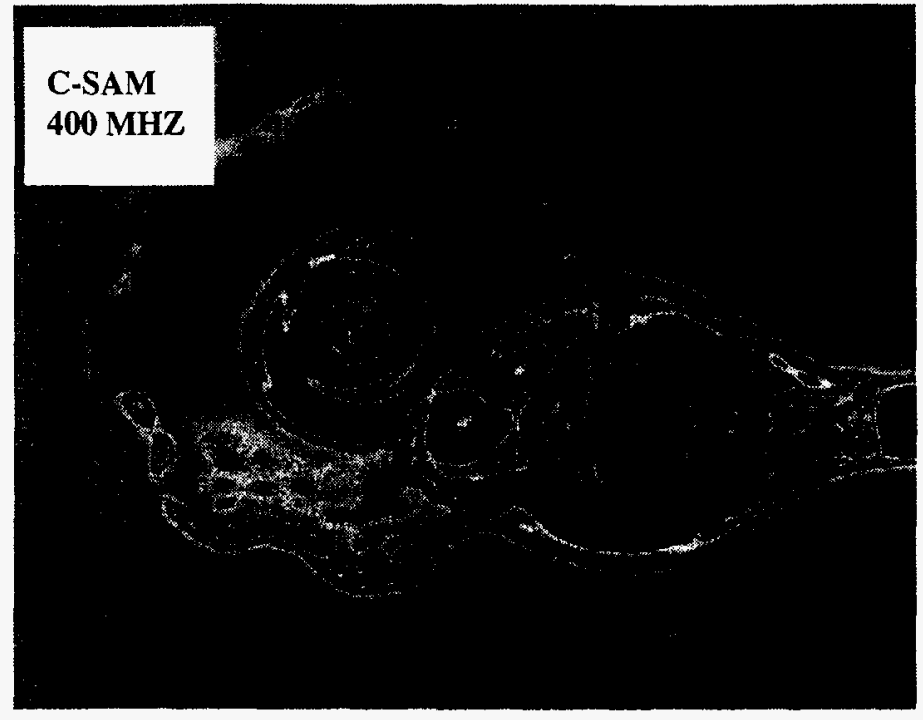

Figure 26. Acoustic microscopy resolves gear, tilt of gear, but no contact.

\section{Failure Modes}

Certain failure modes are catastrophic and have been readily pinpointed by application of standard IC analysis techniques. A more elusive failure mode has been the existence of intermittence in microengines, either as-fabricated, or as a result of brief or extended operation. Degradation in performance of microengines has been attributable to a variety of causes [11]. Examples of failure modes include the following:

Particulate contamination Particulate contamination outside the clean room environment, where performance analysis is conducted, can naturally be expected to have a serious effect on devices where small gaps and tight tolerances are required. Particulate contamination has been a minor problem and has not received the main attention for this study. The particles which receive attention are those which might be internally generated, or present in spite of a clean room environment. An example of a particle was shown in Figure 6.

Fused components A condition which results from overdriving leading to inadvertent contact of structural and electrical members has been observed in springs and in comb fingers. The relatively high voltages employed make arcing possible in these cases, and attachments resembling a weld can result. These attachments are occasionally small enough to be subsequently freed without extensive damage, but more often are permanent.
Sticking Sticking can affect even elements which are not powered. Theories advanced in the literature have attributed aspects of sticking to electrostatic attraction [12] and to moisture adsorption [13]. This work has been less involved with sticking resulting from some artifact of production or storage prior to first operation, and primarily involved with sticking which might occur during operation of a microengine. Some engines show every indication of having a component rigidly adhered to another component or to the substrate, where both components were at the same potential, so fusing due to electrical arcing does not apply.

Intermittence One manifestation of performance degradation is intermittence in the response of a microengine. It might be expected that physical wear would play a role. It is also possible that momentary or reversible sticking could form a point of attachment which is subsequently broken, producing an artifact which acts intermittently and only at a particular point in the rotation of a microengine.

Electrostatic clamping of gears (links) Electrostatic charging of fine MEMS elements, such as electrically isolated gears and links, has been noticed in the SEM and FIB, and charging of comb drives has been induced from laser pulses in their vicinity. Electrostatic attraction has been evident on MEMS elements as they were manipulated with probe tips for the purpose of SEM imaging. Pieces frequently clung to the tips of the probes and were difficult to free.

Three pieces of evidence support a theory of electrostatic clamping at the gear. The first was an observation of a stuck gear while irradiated in the FIB. The number of scans of the gear and links were limited to a few, since the beam is continuously removing material from the surface. The field of view, approximately $100 \mu \mathrm{m}$, was slightly larger than the gear diameter. After the area was subjected to several scans with charge neutralization enabled, the gear suddenly sprung to it's rest position This phenomenon has been observed several times, and occurs only while the charge neutralization is enabled. The second is the observation, using active voltage contrast in the SEM and again in the FIB, of the coincidence of charging/discharging behavior of gears and pin-jointed links with a particular position in the microengine cycle. While this phenomenon is consistent with electrostatic clamping, it should be noted that it would also be observed if the electrical contact to the links and gear were periodic, even in the absence of clamping.

The third observed evidence of clamping occurred while operating microengines during FIB illumination. While illuminating only the gear and a portion of the links, with charge neutralization off, a flex joint engine operated several revolutions and then became clamped. Such gears almost always resume operation with resumption of charge neutralization. The gear in question did not, but was restarted by a slight increase in operating voltage. The gear operated about a minute with charge neutralization off, but the ground plane was exhibiting cyclic voltage contrast. After switching the charge neutralization on, the gear continued rotating and rotated smoothly. The gear then stopped within one rotation of observation after turning off the charge neutralization. Another manifestation of this electrostatic 
charge clamping effect occurred while increasing the magnification with the charge neutralization enabled. The electron flood gun current is constant for a given FIB current, whereas the ionic current density increases with magnification. The rate of charging by the beam at the high magnification overcame the neutralizing effect of the electron beam and an operating engine was stopped. By decreasing the magnification, a lower charging rate was reached where the electron flood gun could neutralize the charge, allowing the engine to rotate. This effect was repeatable.

Many of these events have another factor in common-the intermittent (although usually cyclic) compromise of the ground plane as seen using voltage contrast in both the SEM and the FIB. This could occur from momentary or cyclic contact between a powered comb, and a grounded component or the substrate. This indicates another area where the electrical state of a component of the microengine is not performing as designed.

\section{Conclusions}

Several FA techniques developed for microelectronics have been useful in the FA of MEMS. Evidence of thermal excursions in electrostatic comb drives of degraded microengines have been identified with IR microscopy. Evidence of physical contact of microstructures has been identified optically and with confocal SLM. Voltage contrast in the SEM and in the FIB is an enormous help in determining the relative potential of MEMS elements in a microengine and in an accelerometer. $\mathrm{VC}$ has shown that physical contact does not constitute electrical contact in all cases, and that electrical contact can be intermittent. The demonstration that this is happening periodically during machine operation is important new information. Several observations are consistent with electrostatic clamping of the gear. Some aspects of microengine degradation have eluded detection with existing techniques, such as the inability to observe wear at high magnification in the SEM. New techniques and tools are needed to complete the FA of MEMS and to provide additional information to improve MEMS performance.

\section{Acknowledgments}

The authors would like to thank John Gieske for acoustic microscopy, Jim Rife and Alex Pimentel for FIB analysis, Alan Beattie for help with acoustic emission analysis, and Rich Anderson, Ann Campbell and Ed Cole for helpful comments. This work was supported by the United States Department of Energy under Contract DE-AC04-94AL85000. Sandia is a multiprogram laboratory operated by Sandia Corporation, a Lockheed Martin Company, for the United States Department of Energy.

\section{References}

[1] E.J. Garcia and J.J. Sniegowski, "Surface micromachined microengine," Sensors and Actuators A 48 (1995) 203-214.
[2] S. L. Miller, J. J. Sniegowski, G. LaVigne, and P. J. McWhorter, "Performance tradeoffs for a surface micromachined microengine," Proc. SPIE Micromachined Devices and Components II, Vol. 2882, Austin, October 14-15, pp. 182-191, 1996.

[3] S. L. Miller, J. J. Sniegowski, G. LaVigne, and P. J. McWhorter, "Friction in surface micromachined microengines," Proc. SPIE Smart Electronics and MEMS, Vol. 2722, San Diego, Feb. 28-29, 1996, pp. 197-204.

[4] K.J. Gabriel, F. Behi, R. Mahadevan, and M. Mehregany, "In situ friction and wear measurements in integrated polysilicon mechanisms," Sensors and Actuators, A21-A23 (1990) 184-188. [5] Y.C. Tai and R.S. Muller, "Frictional study of IC-processed micromotors," Sensors and Actuators, A21-A23 (1990) 180-183.

[6] M. Mehregany, S.D. Senturia, and J.H. Lang, "Friction and wear in microfabricated harmonic side-drive motors," Proc. Solid State Sensor and Actuator Workshop, June 4-7, 1990 , Hilton Head Island, South Carolina, pp. 17-22.

[7] A.P. Lee, P.B. Ljung, and A.P. Pisano, 'Polysilicon Micro Vibromotors,' Proceedings of MEMS'92, Travemünde, Germany,Feb. 4-Feb. 7 (1992) 177-182.

[8] S. Suzuki, T. Matsuura, M. Uchizawa, S. Yura, H. Shibata, and H. Fujita, 'Friction and wear studies on lubricants and materials applicable to MEMS,' Proceedings of MEMS'91, Nara, Japan Jan. 30-Feb. 2 (1991) 143-147.

[9] D. M. Tanner, N. M. Smith, D. J. Bowman, W. P. Eaton, and K. A. Peterson, 'First reliability test of a surface micromachined microengine using SHiMMeR,' To be published: Proc. SPIE Micromachined Devices and Components, Vol. 3224, Austin, September 29-30.

[10] A.N. Campbell, J. M. Soden, R. G. Lee, and J. L. Rife, "Electrical biasing and voltage contrast imaging in a focused ion beam system," Proc. Of the 21 st Inter. Symp. For Testing and Failure Analysis, Nov. 6-10, 1995, pp. 33-41.

[11] S. L. Miller, G. LaVigne, M.S. Rodgers, J.J. Sniegowski, J.P. Waters, and P.J. McWhorter, "Routes to failure in rotating MEMS devices experiencing sliding friction To be published: Proc. SPIE Micromachined Devices and Components, Vol. 3224, Austin, September 29-30.

[12] P.R. Scheeper, J.A. Voorthuyzen, W. Olthius and P. Bergveld, "Investigation of attractive forces between PECVD silicon nitride microstructures and an oxidized silicon substrate," Sensors and Actuators A, 30 (1992) pp. 231-239.

[13] C. Linder and N.F. DeRooij, "Investigations on freestanding polysilicon beams in view of their application as transducers," Sensors and Actuators, A21-A23, (1990) pp. 10531059. 
M97007265

Report Number (14) SAND $-97-1733 \mathrm{C}$ CONF -971037-

Publ. Date (11) 199707

Sponsor Code (18) DOE/MA, XF

UC Category (19) U1C-900, DOE IER

DOE 